\title{
Oficina do Código: Um projeto para o ensino e integração de alunos do ensino fundamental e médio na área de tecnologia
}

\author{
Stéphanie Alencar Braga ${ }^{1}$, Caio Raveli F. Barbosa ${ }^{1}$, Priscylla da Silva Tavares ${ }^{1}$, \\ Mikhail José Pires Pedrosa de Oliveira ${ }^{1}$
}

\author{
${ }^{1}$ Instituto Federal de Educação Ciência e Tecnologia do Ceará (IFCE) \\ Av. 13 de Maio, 2081 - Fátima - CEP 60040-531 - Fortaleza - CE - Brasil \\ \{stephanie.abraga, caio.raveli,priscyllatavares,mikhailj.pedrosa\}@gmail.com
}

\begin{abstract}
This paper is a report of the experience acquired in the "Oficina do Código"project: an action of education, integration and formation of Middle and High school students that are included in low-income group of Fortaleza city, located in Ceara state. The project's motivation was the inclusion of these children and teenagers in order to make them capable to contribute to the society growth, improve their creativity, group work and logical and critical thinking.The project is a partnership between the Fortaleza City Government, that sponsored the robotic kits used in class, as well as the others costs and the IFCE (Federal Institute of Ceará), that provided the computer labs. The activities included both theoretical and practical classes aiming to increase the interest of the students in basic projects of the robotics field developed by themselves. In order to help the teaching of programming, electronic circuits and robotics, it was used the open source platform Arduino.
\end{abstract}

Resumo. O presente trabalho é um relato de experiência do projeto Oficina do Código: um projeto que tem como categoria de ação o Ensino, Integração e formação de Crianças do Ensino Fundamental e médio de baixa renda da cidade de Fortaleza-CE. A grande motivação do projeto foi a inclusão dessas crianças para que essas sejam capazes de contribuir para o crescimento da sociedade, estimular a criatividade, trabalho em grupo, pensamento lógico e crítico. O projeto é uma parceria da Prefeitura Municipal de Fortaleza, que custeou os kits de robótica utilizados nas aulas, bem como outros custos com os alunos beneficiados e o IFCE (Instituto Federal do Ceará), que cedeu os laboratórios para as aulas acontecerem. As atividades do Projeto foram feitas através de Aulas Teóricas e Práticas, visando a ambientação e despertar de interesse a projetos básicos de robótica desenvolvidos pelos próprios alunos. Para auxiliar no ensino de programação e prática de eletrônica e robótica foi utilizada a plataforma open source Arduíno.

\section{Introdução}

Segundo França e Amaral (2013), no Brasil, o debate sobre a implantação de um currículo de Computação na educação básica ainda é incipiente. Em 2001, foi criado em Roraima um projeto de lei que viabilizou a implantação da disciplina de Computação no ensino fundamental do $6^{\circ}$ ao $9^{\circ}$ ano e em todos os anos do ensino médio nas escolas da rede pública do Sistema Estadual de Educação. Entretanto, ainda não se observa a publicação 
de nenhum panorama sobre o assunto nos veículos científicos nacionais em que Educação e Computação são tratadas conjuntamente, apesar de outros estados também terem promovido ações voltadas ao ensino de Computação com estudantes do ensino fundamental e médio [França e Amaral 2013].

O contato com a Computação permite o desenvolvimento de diversas capacidades, úteis para qualquer atividade profissional, como é o caso do aprendizado e a aplicação de técnicas para resolver problemas, o contato com a abstração de conceitos e outras diversas competências decorrentes do uso das tecnologias [Scaico et al. 2012]. Para a SBC a Computação deve ser tratada como qualquer outra ciência que auxilie diretamente no desenvolvimento do educando e como consequência, na evolução do país.

Para França et al. (2012), a grande maioria das profissões do século 21 exige uma compreensão de instrumentos ou de conceitos tecnológicos. Profissionais de arte e entretenimento, comunicação, saúde, entre outros, precisam ter conhecimentos tecnológicos. Logo, mesmo para os alunos que não tiveram completa afinidade com estudo e desenvolvimento inicial de tecnologia, tiveram chance de conhecer a forma em que a mesma tem se integrado em outras profissões como ferramentas para agilizar, melhorar e automatizar seus processos específicos.

O incentivo do aprendizado de tecnologia pode beneficiar muitas classes ainda no Brasil. Em seu trabalho Rabelo et al (2015) utilizaram a ferramenta Scratch para produzir recursos interdisciplinares com disciplinas indígenas estimulando o pensamento computacional através destas atividades, integrando programação, arte e cultura e língua Tupi. Já em Maliuk (2009), o autor mostrou a robótica no ensino de matemática para alunos do nono ano da escola EMEF José Mariano Beck em Porto Alegre. O objetivo era melhorar o rendimento dos alunos na disciplina de matemática e, posteriormente, despertar o interesse deles no mundo tecnológico.

No presente trabalho é apresentado o projeto Oficina do Código, que foi planejado para introduzir alunos de escolas públicas da cidade de Fortaleza no meio da Computação e Tecnologia, apresentando aos alunos desde aspectos teóricos de Hardware e Software até o desenvolvimento prático de um carrinho microcontrolado utilizando a plataforma open source Arduíno. A ideia fundamental do projeto é despertar o interesse dos jovens no mundo tecnológico de maneira profissional, visto que, no caso do Brasil, o aumento na demanda por profissionais de tecnologia reforça a necessidade de atraí-los desde cedo [Meneses et al. 2015].

O trabalho está apresentado da seguinte maneira: a seção 2 descreve o projeto e o quê o motivou, a seção 3 apresenta a plataforma Arduíno no auxílio da educação, a seção 4 descreve as etapas de planejamento e metodologia, a seção 5 as fases de execução do projeto, a seção 6 apresenta os resultados e análise e, por fim, a seção 7 apresenta as considerações finais.

\section{Motivação e Descrição do Projeto}

A Sociedade Brasileira de Computação - SBC atua e defende o ensino da Computação enquanto ciência desde a educação básica, pois segundo a mesma, o domínio da Computação e das tecnologias da informação é fundamental e estratégico para o desenvolvimento social e econômico mundial [Silva et al. 2015]. Nesse contexto, a grande 
motivação do projeto foi a inclusão de crianças do Ensino Fundamental de baixa renda para que essas sejam capazes de contribuir para crescimento da sociedade, estimular a criatividade, trabalho em grupo, pensamento lógico e crítico.

O projeto Oficina do Código aprovado no edital 04/2014 da prefeitura municipal de Fortaleza como um dos projetos do Programa "Nossa ação muda a cidade", buscava ensinar às crianças das escolas públicas de Ensino Fundamental $\left(5^{\circ}\right.$ a $9^{\circ}$ ano $)$ de Fortaleza, a lógica e linguagem básica de programação e fundamentos de eletrônica atrelando, posteriormente, à Robótica. A equipe do projeto era composta por 4 integrantes, dois coordenadores e 2 professores, que visavam proporcionar conhecimento básico nessas áreas citadas, para despertar a curiosidade e capacitar alunos de baixa renda na área da Tecnologia.

\section{A Plataforma Arduíno e a educação}

O fato do projeto Oficina do Código ter como objetivo principal a introdução de conceitos voltados à computação e tecnologia para iniciantes, foi escolhida a plataforma microcontrolada Arduíno. Essa plataforma de prototipagem serviu de auxilio no ensino de crianças, adolescentes, jovens ou adultos, por se tratar de uma ferramenta de cógigo aberto (open source), de baixo custo e de fácil utilização [Perez et al. 2013]. Com essa ferramenta, foi possível mostrar na prática conceitos de programação e eletrônica.

O Arduíno teve seu início comercialmente em 2005 no Interaction Design Institute na cidade de Ivrea, na Itália, desenvolvido pelo professor Massimo Banzi e pelo pesquisador da Universidade de Malmo, na Suécia, chamado David Cuartielles. Anteriormente, o Arduíno foi desenvolvido como recurso auxiliar no ensino dos estudantes. Porém, como os produtos no mercado eram caros e difíceis de usar, Banzi e Cuartielles desenvolveram um microcontrolador com as principais exigências a um preço baixo e com uma facilidade de uso de tal forma que qualquer pessoa pudesse utilizá-lo [Evans et al. 2013].

A plataforma Arduíno tem outras facilidades que a torna bastante flexível para inseri-la no meio educacional quando o ensino é relacionado à tecnologia. Esta possui em seu hardware uma interface de comunicação USB (Universal Serial Bus) para acessar de forma mais simples o seu microcontrolador, tem um próprio ambiente de desenvolvimento integrado (IDE), do inglês Integrated Developed Environment, que é apresentado de forma bem intuitiva, utilizando uma linguagem de programação baseada nas linguagens $\mathrm{C}$ e $\mathrm{C}++$. Assim, torna-se descomplicado fazer recepção e envio de sinais analógicos/digitais para componentes como sensores, atuadores, leds, motores, entre outros [Kalil et al. 2013].

\section{Etapas de Planejamento e Metodologia}

Após a aprovação do projeto Oficina do Código, iniciaram-se as etapas de planejamento, antes do mesmo começar a ser executado. Nas seções subsequentes estão descritas essas etapas.

\subsection{Escolha e Aquisição do Material Didático}

Após o recebimento dos recursos financeiros para o projeto, foi iniciada a compra do material necessário para as aulas, para que estes chegassem a tempo da realização do curso. Foi realizada uma pesquisa de preços, pois o fomentador do projeto exigia 3 orçamentos, 
dos quais seria escolhido o com preço mais acessível. Devido as limitações no orçamento, foram comprados os materiais da Tabela 1.

Tabela 1. Materiais utilizados nas aulas
\begin{tabular}{|l|l|}
\hline Material & Quantidade \\
\hline Kit de Desenvolvimento Arduíno Uno & 8 \\
\hline Kit de componentes eletrônicos & 8 \\
\hline Chassi com rodas e motores & 8 \\
\hline Multimetros & 4 \\
\hline Baterias para alimentação dos kit de robótica & 8 \\
\hline
\end{tabular}

\subsection{Inscrições e Seleção dos alunos}

Depois de um consenso entre a equipe executora, foi tomada a decisão de que os alunos que seriam selecionados para participar do projeto deviam obedecer aos seguintes critérios:

- Os alunos seriam de escolas diferentes, ou seja, não escolheu-se uma escola específica para selecionar os alunos;

- Os alunos teriam que comprovar baixa renda, por meio de documentos;

- Tinham que estar cursando entre o $6^{\circ}$ ano do ensino fundamental e $3^{\circ}$ ano do ensino médio;

Os alunos inscritos foram selecionados por meio da análise de notas. Primeiro analisou-se os que tinham melhores notas em matemática. Em caso de empate, os que tinham as melhores notas em português. Permanecendo, a média geral de todas as disciplinas.

O projeto foi divulgado por meio de cartazes, redes sociais, rádios locais e pessoalmente em algumas escolas, por um período de aproximadamente dois meses. Ao todo, se inscreveram 54 alunos ( 32 do sexo masculino e 22 do sexo feminino) e foram selecionados 17. Dos selecionados apenas 2 estavam no ensino médio, o restante ainda cursava o ensino fundamental II. Os alunos selecionados não tinham conhecimento prévio ou alguma experiência sobre programação/robótica.

O perfil da turma, após serem selecionados os alunos, está ilustrado na Figura 1.

\subsection{Ementa do Curso}

Como o objetivo do curso era aliar conhecimentos de computação/programação e eletrônica, a escolha da ementa buscou inserir conteúdos básicos de computação e eletricidade nas primeiras aulas, o que daria suporte às aulas práticas de programação com a plataforma Arduíno. A Tabela 2 mostra como ficou a ementa inicial do curso.

\section{Execução do Projeto}

Após a turma selecionada, ementa definida e materiais adquiridos, o projeto começou a ser executado. Houve uma parceria com o Instituto Federal do Ceará (IFCE) - Campus Fortaleza, que cedeu o espaço de seus laboratórios para que as aulas fossem ministradas. 


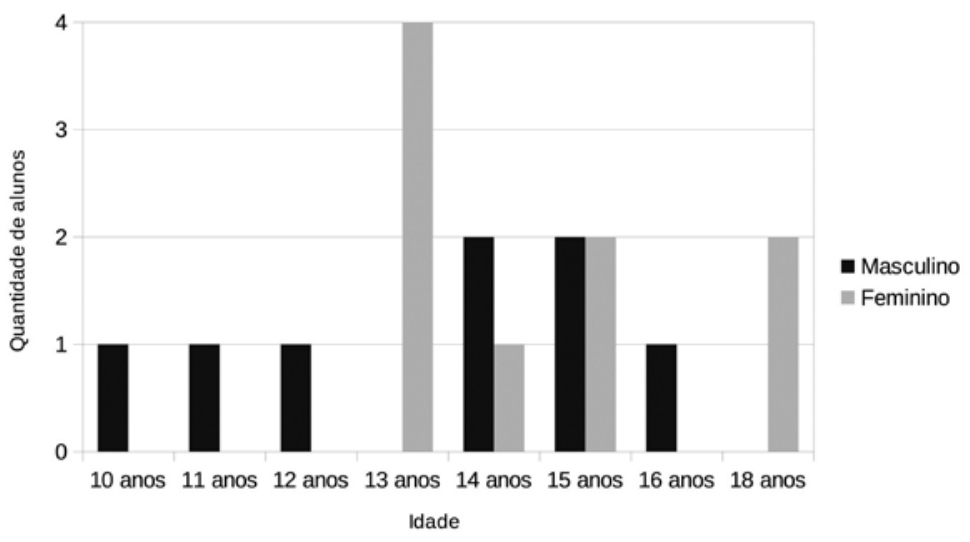

Figura 1. Perfil dos Alunos

Tabela 2. Ementa do Curso

\begin{tabular}{|c|c|}
\hline & Conteúdo \\
\hline Aula 1 & $\begin{array}{l}\text { - Apresentação Inicial: Explicar o projeto, o que irá ser estudado. } \\
\text { - Mostrar exemplos de Tecnologias existentes. } \\
\text { - Apresentar as ferramentas que serão utilizadas (Placas, exemplos de } \\
\text { programação, etc). } \\
\text { - Apresentação de vídeos e exemplos práticos, visando motivar as } \\
\text { crianças. }\end{array}$ \\
\hline Aula 2 & $\begin{array}{l}\text { - Explicar o conceito de eletricidade: Corrente, Tensão contínua e Alter- } \\
\text { nada. } \\
\text { - Apresentar alguns componentes eletrônicos e mostrar o funcionamento } \\
\text { de alguns: resistor, leds, fontes de bancada. } \\
\text { - Explicar conceitos básicos de eletrônica digital. }\end{array}$ \\
\hline Aula 3 & $\begin{array}{l}\text { - Mostrar aplicações com todos os conceitos mencionados e introduzir o } \\
\text { conceito de microcontroladores. } \\
\text { - Prática com o multímetro: mostrar o funcionamento e realizar algumas } \\
\text { medições. }\end{array}$ \\
\hline Aula 4 & $\begin{array}{l}\text { - Apresentação do que é programação. } \\
\text { - Práticas com o site "Hora do Código". }\end{array}$ \\
\hline Aula 5 & $\begin{array}{l}\text { - Conceitos de programação. } \\
\text { - Práticas. }\end{array}$ \\
\hline Aula 6 & $\begin{array}{l}\text { - Conceitos de programação. } \\
\text { - Práticas. }\end{array}$ \\
\hline Aula 7 & $\begin{array}{l}\text { - Conceitos de programação. } \\
\text { - Práticas. }\end{array}$ \\
\hline Aula 8 & $\begin{array}{l}\text { - Apresentação da plataforma Arduíno. } \\
\text { - Identificação dos conceitos estudados utilizando Arduíno. } \\
\text { - Práticas. }\end{array}$ \\
\hline Aula 9 & $\begin{array}{l}\text { - Introdução à robótica. } \\
\text { - Definição do projeto prático final. } \\
\text { - Práticas. }\end{array}$ \\
\hline Aula 10 & - Execução da Projeto final com os kits de Robótica. \\
\hline
\end{tabular}




\subsection{Aulas}

O projeto foi desenvolvido por meio de aulas apenas teóricas e aulas em que houve uma parte teórica e prática. As aulas aconteciam aos sábados e tinham a duração de 4 horas. Iniciavam às $8 \mathrm{~h}$ e terminavam às $12 \mathrm{~h}$, com pausa de 15 minutos para lanche.

No laboratório havia computadores suficientes para que os alunos ficassem no máximo aos pares. Havia também um data show para a utilização de slides e ilustrações na hora da aula. O conteúdo era ministrado pelos dois professores do projeto, contando também com o auxílio dos dois coordenadores. A Figura 2 mostra uma foto registrada da primeira aula do projeto.

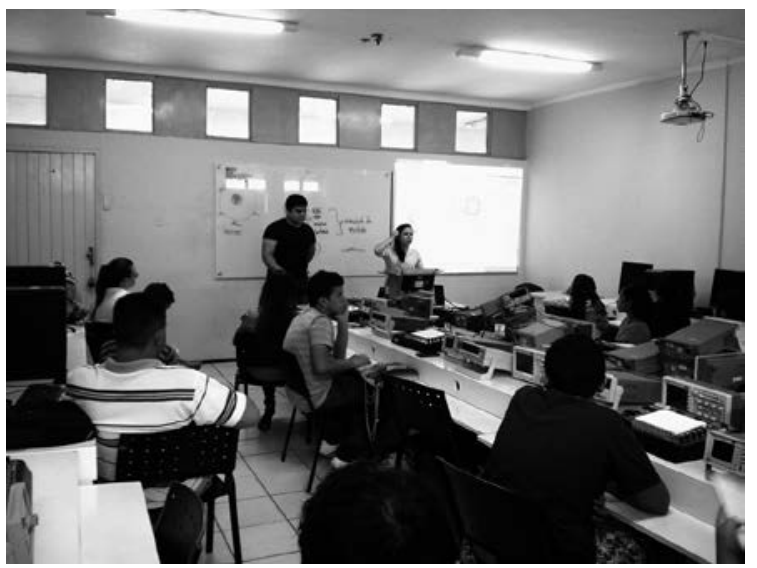

Figura 2. Foto da primeira aula

\subsection{Mudanças na ementa}

Apesar de ter sido planejada uma ementa, na prática a mesma foi modificada e adaptada, de acordo com as necessidades observadas em cada aula.

Até a aula 4 não houve mudanças, mas a partir da aula 5 a ementa mostrada na Tabela 2 foi praticamente toda modificada. Na aula 5 já foi apresentada a plataforma Arduíno, pois na aula 4 os alunos conseguiram assimilar bem alguns conceitos básicos de programação, como o que são variáveis, tipos de variáveis, declaração, etc. Como os alunos iriam praticar a programação utilizando a plataforma Arduíno, decidiu-se começar as práticas diretamente na plataforma. As práticas foram definidas e inseridas nas aulas, pois ao final iriam ser integradas ao projeto final com o carrinho. A Tabela 3 mostra as mudanças que ocorreram na ementa a partir da aula 5.

\subsection{Projeto prático}

No decorrer das aulas, foi estabelecido o objetivo de, ao fim do curso, executar um projeto prático utilizando o carrinho e os sensores adquiridos. A partir da aula 7, as práticas realizadas serviram para compor o projeto final: "Botar o carrinho pra andar".

Ao fim, os próprios alunos realizaram a montagem de 8 unidades do projeto final, com o auxílio dos professores caso tivessem alguma dúvida. Os materiais utilizados em cada um foram os seguintes: carrinho, 2 motores CC, Arduíno Uno, sensor ultrassônico, 4 pilhas. O código final do projeto foi uma junção dos códigos que iam sendo construídos nas aulas práticas. A Figura 3 ilustra a versão final de um dos carrinhos montados. 
Tabela 3. Ementa modificada

\begin{tabular}{|l|l|}
\hline & Conteúdo \\
\hline Aula 5 & • Apresentação da plataforma Arduíno e programações inicias. \\
\hline Aula 6 & • Práticas iniciais utilizando a plataforma Arduíno: Led + botão. \\
\hline Aula 7 & $\begin{array}{l}\text { - Arduíno e sensores: estudo do sensor ultrassônico. } \\
\text { - Prática com o sensor ultrassônico. }\end{array}$ \\
\hline Aula 8 & • Montagem do robô. \\
\hline Aula 9 & • Prática: Arduíno e motor CC junto com o carrinho. \\
\hline Aula 10 & $\begin{array}{l}\text { - Prática final: "Botando o carrinho pra andar". Junção do sensor ul- } \\
\text { trassônico + motor. }\end{array}$ \\
\hline
\end{tabular}

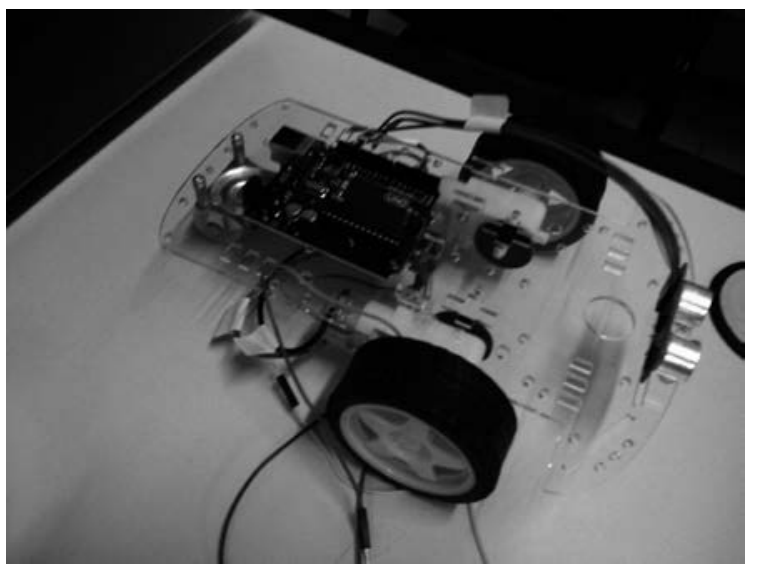

Figura 3. Versão final de um dos carrinhos montados

\section{Análise dos Resultados}

Durante o período de realização do projeto, aproximadamente 3 meses, foi possível observar, comprovar e aprender muitas coisas. Com relação à equipe executora do projeto (professores e coordenadores), a experiência foi de grande valia e a oportunidade de lidar com um público que ainda não se tinha trabalhado foi de grande importância e aprendizado. Isso pode ser observado no fato da mudança da ementa do curso. As ações planejadas nem sempre se realizavam como se esperava, levando a equipe a mudar um pouco a metodologia.

Com relação aos alunos, pode-se comprovar que o número de alunos que concluíram o curso foi inferior ao número de alunos que iniciaram. Apesar do esforço da equipe em motivá-los, ainda houve 2 desistências, totalizando 15 alunos concludentes. Uma das desistentes informou que desistiu por questões de dificuldade de locomoção até o IFCE e o outro não informou a respeito. Quanto aos outros houve boa frequência, apenas 1 aluno faltou 3 vezes, 4 alunos faltaram 2 vezes e 2 alunos faltaram 1 vez. A Figura 4 mostra os alunos recebendo o certificado de conclusão no fim do curso.

Mesmo o curso apresentando boa frequência, o impacto que a falta de um aluno causa na aula é significante, já que a turma é pequena. Principalmente nas aulas inicias, onde abordava-se mais a teoria, era necessário no começo da aula, fazer uma espécie de revisão do conteúdo da aula anterior. Porém, apesar de atrasar um pouco a aula servia para os alunos que não faltaram relembrarem o conteúdo e assim assimilarem melhor. 
Quanto ao interesse e desempenho dos alunos durante as aulas, foi possível observar que havia um interesse maior pelas aulas práticas, por exemplo utilizando o multímetro, os componentes eletrônicos e a placa arduíno e até mesmo com programação. Na hora de montar a parte mecânica do carrinho os alunos tiveram um desempenho surpreendente, a maioria sendo capaz de apenas ler o manual e montar corretamente. Também se mostravam bastante pró-ativos com relação aos equipamentos, demonstrando total interesse pelas coisas novas que estavam vivenciando.

Em relação à interação dos alunos com a linguagem de programação, os resultados foram bastante satisfatórios. A partir da aula 4 já foram introduzidos os primeiros conceitos com o desafio "Programação com Anna e Elsa", disponível em [A Hora do Código ]. Os alunos tiveram ótimo desempenho no desafio e conseguiram resolver rapidamente, mostrando-se bastante interessados.

Já quando foi introduzida a plataforma Arduíno, os alunos tiveram um pouco de dificuldade, sendo necessário relembrá-los quase sempre as funções. A maior dificuldade encontrada foi devido ao idioma (inglês) das funções utilizadas no Arduíno, pois os alunos em sua grande maioria não sabiam inglês ou sabiam muito pouco. Esse fato dificultou a interação dos alunos quando iam implementar os códigos, pois eles tiveram dificuldade de assimilar o nome das funções e a relacionar o nome à sua real função.

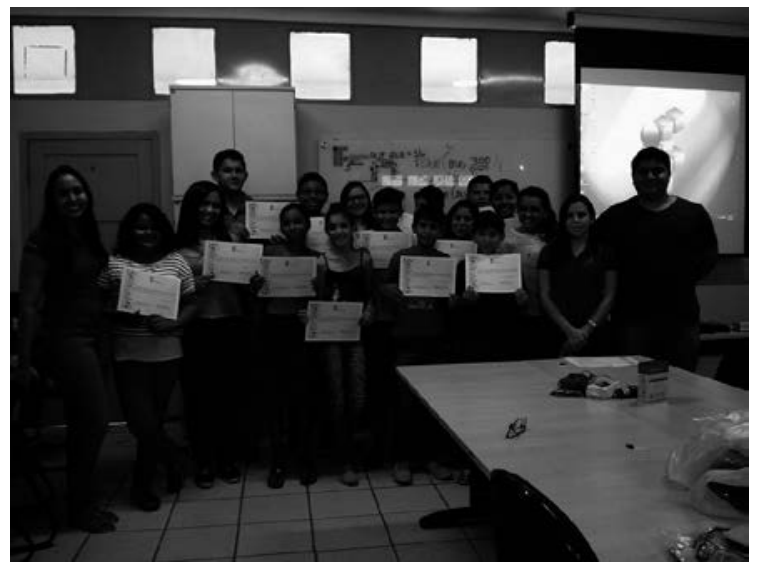

Figura 4. Entrega dos certificados aos alunos no fim do curso

\section{Considerações Finais}

Este artigo apresentou um relato de experiência descrevendo as etapas de desenvolvimento e resultados obtidos com o projeto Oficina do Código. Pelos resultados apresentados, apesar de algumas dificuldades encontradas, o objetivo do projeto foi atingido, pois os alunos mostraram-se bastante interessados pela área de computação. Alguns mostraram-se bastante interessados pela plataforma Arduíno, e outros disseram que queriam seguir carreira nessa área de computação.

Pelo perfil da turma foi possível comprovar que os alunos não tinham conhecimento, ou tinham muito pouco sobre programação/robótica. Os assuntos ensinados eram novidade para a grande maioria, apenas 1 dos 17 alunos já tinha participado de uma iniciativa de ensino a robótica na escola onde estudava. Os demais ainda não tinham vivenciado esse tipo de experiência, nem projetos nem na escola onde estudavam. Isso mostra que as escolas públicas de Fortaleza ainda têm muito a avançar nessa área. 


\section{Agradecimentos}

A Prefeitura Municipal de Fortaleza e ao Banco Interamericano de Desenvolvimento (BID) pelo financiamento do projeto, ao IFCE por ter cedido o espaço e equipamentos dos laboratórios e ao professor Edson Almeida, chefe do departamento de Telemática do IFCE, por se mostrar bastante prestativo às necessidades logísticas do projeto.

\section{Referências}

A Hora do Código. Programação com Anna e Elsa. https: / / studio. code.org/ s/frozen/stage/1/puzzle/1. Accessado em março de 2016.

Evans, M., Noble, J., and Hochenbaum, J. (2013). Arduíno in Action. Manning Publications. New York.

França, R. S. and Amaral, H. J. C. (2013). Ensino de Computação na Educação Básica no Brasil: Um Mapeamento Sistemático. In XXI Workshop Sobre Educação em Computação (WEI), Maceió - AL.

França, R. S., da Silva, W. C., and do Amaral, H. J. C. (2012). Ensino de Ciência da Computação na Educação Básica: Experiências, Desafios e Possibilidades. In $X X$ Workshop Sobre Educação em Computação (WEI), Curitiba - PR.

Kalil, F., Hernandez, H., Antunez, M. F., Oliveira, K., Ferronato, N., and dos Santos, M. R. (2013). Promovendo a robótica educacional para estudantes do ensino médio público do Brasil. In Nuevas Ideas en Informática Educativa TISE. Porto Alegre - RS.

Maliuk, K. D. (2009). Robótica educacional como cenário investigativo nas aulas de matemática. In Dissertação (Mestrado em ensino de matemática) - Instituto de Matemática. Universidade Federal do Rio Grande do Sul. Porto Alegre.

Meneses, L. F., Mai, L. F. F., Rosario, J., Oliveira, E., and Gomes, R. L. (2015). IntroComp: Atraindo Alunos do Ensino Médio para uma Instigante Experiência com a Programação. In XXIII Workshop Sobre Educação em Computação (WEI), Recife PE.

Perez, A. L. F., Darós, R., Puntel, F. E., and Vargas, S. R. (2013). Uso da Plataforma Arduino para o Ensino e o Aprendizado de Robótica. In International Conference on Interactive Computer aided Blended Learning.

Rabelo, H. M., de Oliveira, W. K. F., Santos, L. L., de Araújo, A. L. S. O., and Souza, F. V. C. (2015). Scratch na produção de recursos interdisciplinares com disciplinas indígenas. In XXIII Workshop Sobre Educação em Computação (WEI), Recife - PE.

Scaico, P. D., Corlett, E. F., Paiva, L. F., Raposo, E. H. S., and Alencar, Y. (2012). Relato da Utilização de uma Metodologia de Trabalho para o Ensino de Ciência da Computação no Ensino Médio. In XVIII Workshop de Informática na Escola, Rio de Janeiro- RJ.

Silva, S. F., Barbosa, A. F., Souza, A. A., Silva, E. G., Oliveira, M. L. S., Silva, S. R., and Santos, W. O. (2015). Relato de Experiência de Ensino de Computação no Ensino Fundamental em Estágio Supervisionado da Universidade de Pernambuco no Campus Garanhuns. In XXIII Workshop Sobre Educação em Computação (WEI), Recife - PE. 